\title{
Three-dimensional magnetic reconnection regimes: A review
}

\author{
D. I. Pontin*
}

\begin{abstract}
The magnetic field in many astrophysical plasmas - such as the Solar corona and Earth's magnetosphere - has been shown to have a highly complex, three-dimensional structure. Recent advances in theory and computational simulations have shown that reconnection in these fields also has a threedimensional nature, in contrast to the widely used two-dimensional (or 2.5-dimensional) models. Here we discuss the underlying theory of three-dimensional magnetic reconnection. We also review a selection of new models that illustrate the current state of the art, as well as highlighting the complexity of energy release processes mediated by reconnection in complicated three-dimensional magnetic fields.
\end{abstract}

\section{Introduction}

Magnetic reconnection is a fundamental process that is ubiquitous in astrophysical plasmas. It facilitates the release of energy stored in the magnetic field by permitting a change in the magnetic topology in an almost ideal plasma. As such, reconnection is universally accepted to be a key ingredient in the behaviour of many astrophysical plasmas, including the interiors and atmospheres of stars such as the Sun, planetary magnetospheres, accretion disks, and pulsar magnetospheres.

Much of the literature on reconnection focusses on the two-dimensional problem, due to the theoretical and computational simplifications that this allows. However, it is now becoming clear that magnetic reconnection in an even weakly three-dimensional (3D) setting is crucially different from the planar $2 \mathrm{D}$ case. In this article, we review recent advances in three-dimensional reconnection theory, and the complex picture that is emerging of the possible regimes of reconnection in 3D. This review is by necessity limited and misses a number of important facets of reconnection research. Complementary reviews include those by Priest and Forbes (2000); Biskamp (2000); Zweibel and Yamada (2009); Yamada et al. (2010). In Section 2 we introduce some key measures and features of magnetic field structure in 3D that are crucial to understanding where and how reconnection operates in 3D, while in Section 3 we discuss some fundamental differences between 2D and 3D reconnection. In Sections 446 we review the current picture of the various different 3D reconnection regimes, and in Section 7 we touch briefly on recent results from large-scale numerical simulations and observations. We finish with a summary in Section 8 .

\section{Magnetic topological and geometrical structures}

Recent observations and analysis are revealing the complex structure of the magnetic field in astrophysical plasmas. These studies have naturally focussed on solar system plasmas - in particular the solar atmosphere and Earth's magnetosphere - because these are environments that we can observe with relatively high spatial resolution. However, it is very likely that this rich structure is present also in other astrophysical bodies.

One crucial step in understanding the behaviour of astrophysical plasmas is to determine just where magnetic reconnection may occur, and therefore what are the likely locations of energy release. Whether

\footnotetext{
*Division of Mathematics, University of Dundee, Dundee, U.K.; e-mail: dpontin@maths.dundee.ac.uk
} 

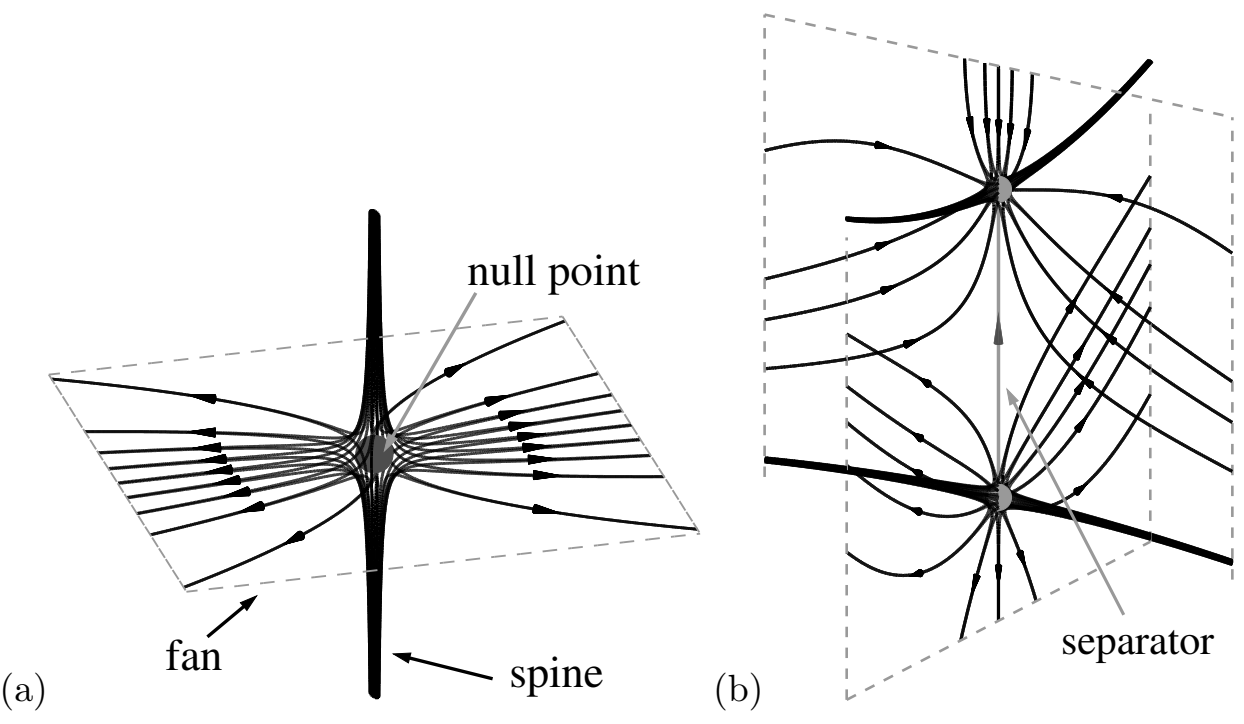

Figure 1: Potential magnetic field line structure in the vicinity of (a) an isolated 3D null point, and (b) a generic fan-fan separator

in a collisional or collisionless plasma, magnetic reconnection requires the presence of a current sheet. So in order to determine the locations where reconnection can facilitate the release of energy in the plasma, we must understand where current sheets form. (Note: in this article we use the term 'current sheet' to refer to any intense, localised current layer - rather than, as is sometimes the case, reserving the term for singular current structures.) In two dimensions, it is well established that reconnection occurs at magnetic X-points, which are prone to collapse to form current layers. However, with the loss of two dimensional symmetry, the number of proposed sites of current sheet formation and reconnection is greatly increased. These proposed sites can be broadly divided into two classes, being either topological or geometrical features of the magnetic field. A topological feature is preserved by an arbitrary smooth deformation of the magnetic field, while we will refer to any property that is not preserved by all such deformations as a geometrical feature.

One natural extension from the 2D X-point collapse picture is the idea of current sheet formation at $3 \mathrm{D}$ null points - points in space at which the magnetic field strength falls to zero. Null points are topological features of the magnetic field, and their structure has been studied by a number of authors (Fukao et al., 1975; Lau and Finn, 1990; Parnell et al., 1996) - a typical configuration is shown in Figure 1(a). Since $\nabla \cdot \mathbf{B}=0$, magnetic null points must be of hyperbolic type. Their structure is characterised by a pair of field lines that asymptotically approach (or recede from) the null from opposite directions, forming the spine (or $\gamma$-line) of the null, while field lines recede from (or approach) the null in a surface known as the fan (or $\Sigma$-) plane. This surface is a separatrix surface - it separates topologically distinct volumes of magnetic flux.

Another proposed site of current sheet formation in 3D is a separator line - a field line that runs from one null point to another. In dynamical systems theory this would be called a heteroclinic orbit. Such a field line is defined by the transverse intersection of the fan planes of the two nulls (Figure 1(b)) and is therefore topologically stable. Nulls and separators were first suggested as possible sites of current sheet formation due to the discontinuous jump in field line connectivity at their associated separatrix structures. Therefore, if one considers the implications of an ideal flow across these separatrix structures in a kinematic model, singularities in the electric field result (Lau and Finn, 1990; Priest and Titov, 1996). It is worth noting that these considerations apply equally to closed magnetic field lines, as discussed by Lau and Finn (1990). These closed field lines play a crucial role in laboratory plasmas - however, since 
the magnetic field in astrophysical plasmas is usually considered to be anchored at the surface of the neighbouring star or planet, closed field lines do not typically take centre stage here.

A question that naturally arises is: do such null points and separators exist in space plasmas? New observations and theoretical studies suggest that they are abundant. Recent analysis of in-situ observations by the Cluster mission show the presence of both single nulls and collections of nulls located in the current sheet of the Earth's magnetotail (e.g. Xiao et al., 2006; Deng et al., 2009). Furthermore, the standard model of the magnetosphere contains two null points in the cusp regions joined by separator lines, while clusters of nulls (expected to be joined by separators) have been found in the global magnetosphere simulations of Dorelli et al. (2007). Turning our attention to the solar atmosphere, the lack of magnetic field measurements in the corona renders direct detection impossible at present. However, increasingly detailed magnetograms at the level of the photosphere permit extrapolation of the field into the corona. Such extrapolations in quiet sun regions show an abundance of 3D nulls to be present, with a high density at chromospheric levels, falling off exponentially with height (e.g. Régnier et al., 2008, Longcope and Parnell, 2009). Nulls have also been inferred to be present in many flaring and erupting active regions, as discussed later.

Nulls and separators are, however, not the end of the story. In 3D, current sheet formation and magnetic reconnection may also occur in the absence of nulls. However, one still requires some mechanism to generate intense current layers in the plasma. In the case of the Earth's magnetosphere, the system is being continually driven by the incident solar wind. This leads to large-scale current structures both at the dayside magnetopause and in the magnetotail (though what triggers these current layers to thin resulting in the onset of fast reconnection is still an open question). In the solar atmosphere, however, the driving of the system is less direct, occurring at the photospheric footpoints, and the question of how this photospheric driving maps eventually to the creation of coronal current sheets is not straightforward to answer. There are certain magnetic field structures that appear to encourage the formation of current layers. One such topological structure is a region of braided magnetic field - in which field lines are non-trivially linked with one another. A recent series of papers has demonstrated that the small scales associated with the field line mapping in braided fields may lead to a loss of equilibrium, leading to the formation of multiple small-scale current layers (Wilmot-Smith et al., 2009a b, 2010; Pontin et al. 2011). However, current layers may also form in topologically simple fields, for example as the result of some ideal instability. Browning et al. (2008) and Hood et al. (2009) have followed the evolution of a kink-unstable flux tube in resistive MHD simulations and found a complex array of current layers to form during the subsequent relaxation.

The structure of a magnetic field may in general be characterised by the mapping generated by the connectivity of magnetic field lines within the domain. In the absence of null points this mapping is continuous. However, it has been proposed that if sufficiently strong gradients are present in this mapping then intense current layers will in general form when the field is perturbed by plasma motions. (e.g. Longcope and Strauss, 1994; Priest and Démoulin, 1995). These gradients in the connectivity are characterised by the so-called squashing factor, $Q$ (Titov et al., 2002; Titov, 2007), and regions with high values of $Q$ are usually termed quasi-separatrix layers (or QSLs). The name stems from the fact that a true separatrix surface may be considered as the limit obtained when a QSL approaches zero thickness and infinite $Q$. For a detailed review of these ideas, see Démoulin (2006).

A further proposition, originally put forward to explain the heating of the solar corona by Parker (1972), is that any generic footpoint motion will naturally lead to current sheet formation in the corona. This has been investigated by a number of authors using various numerical simulations, without any clear consensus being reached about the nature of the current structures formed (in particular whether they are singular in the ideal limit) or whether they could account for the heating of the solar corona at realistic coronal plasma parameters (e.g. van Ballegooijen, 1985; Longcope and Strauss, 1994; Hendrix and van Hoven, 1996; Galsgaard and Nordlund, 1996; Ng and Bhattacharjee, 1998; Rappazzo et al., 2008).

Here we have given a short (and certainly not exhaustive) introduction describing some possible 
sites and mechanisms of current sheet formation in 3D magnetic fields. The main focus of this article, however, is not on where magnetic reconnection may take place in $3 \mathrm{D}$, but rather on the properties of the reconnection process when it does take place.

\section{Fundamental properties of 3D reconnection}

Under what conditions does magnetic reconnection occur in 3D? To answer this question we first require a definition of reconnection in 3D. The most general approach, the one that we follow here, was put forward by Schindler et al. (1988); Hesse and Schindler (1988) in the framework of general magnetic reconnection. Within this framework magnetic reconnection is defined by a breakdown of magnetic field line and flux conservation, or in other words a breakdown in the magnetic connection between plasma elements. This was shown to occur in three dimensions in general when a component of the electric field parallel to the magnetic field $\left(E_{\|}\right)$is spatially localised in all three dimensions. The change of connectivity, or reconnection rate, is quantified by the maximal value (over all field lines) of

$$
\Phi=\int E_{\|} d s
$$

where the integral is performed along magnetic field lines from one side of the diffusion region (region within which $E_{\|} \neq 0$ ) to the other.

It is now being appreciated that the fundamental properties of $3 \mathrm{D}$ reconnection are crucially different from the simplified 2D picture. These new properties can be understood by considering the implications of the following equation

$$
\frac{\partial \mathbf{B}}{\partial t}-\nabla \times(\mathbf{w} \times \mathbf{B})=\mathbf{0}
$$

which describes the ideal evolution of a magnetic field, where $\mathbf{w}$ is a flux-conserving velocity or flux transport velocity (which in ideal MHD is simply the fluid velocity $\mathbf{v}$ ). If for a given magnetic field evolution a smooth flow $\mathbf{w}$ exists then the magnetic flux is frozen into the flow $\mathbf{w}$, and the topology of the magnetic field is preserved - this being guaranteed by the condition that $\mathbf{w}$ be smooth and continuous. (Note that strictly speaking, the topology is still preserved if the right-hand side of Equation (2) is a non-zero term parallel to $\mathbf{B}$, say $\lambda \mathbf{B}$ where $\lambda$ is some scalar field - see Hornig and Schindler (1996).) Using Faraday's law and 'uncurling' Equation (2),

$$
\mathbf{E}+\mathbf{w} \times \mathbf{B}=\mathbf{R}, \quad \mathbf{R}=\nabla \Psi
$$

where $\Psi$ is a free function. In $2 \mathrm{D}$, the conditions on the existence of $\mathbf{w}$ are straightforward. Assuming that the magnetic and plasma flow fields $(\mathbf{B}$ and $\mathbf{v})$ are $2 \mathrm{D}$, then we must have $\mathbf{E} \cdot \mathbf{B}=0$ and $\mathbf{R} \cdot \mathbf{B}=0$, so we can write $\mathbf{R}=\delta \mathbf{w} \times \mathbf{B}$, say, which leads to

$$
\mathbf{E}+(\mathbf{w}-\delta \mathbf{w}) \times \mathbf{B}=\mathbf{0} .
$$

So in $2 \mathrm{D}$ a flux transporting flow exists everywhere, and is given by

$$
\mathbf{w}=\delta \mathbf{w}+\frac{\mathbf{E} \times \mathbf{B}}{B^{2}}
$$

(see Hornig (2001) for a more detailed exposition). It is clear that this velocity is smooth everywhere except at null points (at which $\mathbf{B}=\mathbf{0}$ ) where it is singular unless $\mathbf{E}=\mathbf{0}$ there. The above derivation of an explicit expression for $\mathbf{w}$ relies crucially on the condition that $\mathbf{E} \cdot \mathbf{B}=0$. However, in $3 \mathrm{D}$ reconnection by definition $\mathbf{E} \cdot \mathbf{B} \neq 0$ (see above), and the conditions under which magnetic topology conservation, field line conservation, and magnetic flux conservation hold are much more subtle - the reader is referred to the papers by Schindler et al. (1988); Hornig and Schindler (1996); Hornig (2001, 2007a b). 

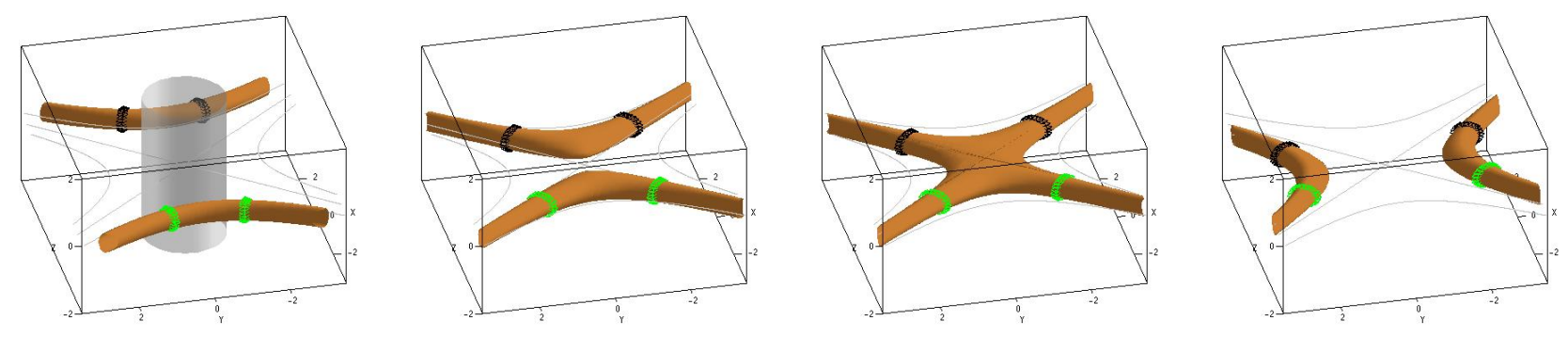

Figure 2: Reconnection of two representative flux tubes in the magnetic field $\mathbf{B}=\left(y, k^{2} x, 0\right)$, with $k=1.2$. A localised diffusion region (shaded surface) is present around the $\mathrm{X}$-point.

As demonstrated above, in 2D a smooth flux transporting velocity exists everywhere with the possible exception of magnetic nulls. The magnetic null may be of O-type or X-type: for an O-point with non-zero electric field there is annihilation (or creation) of magnetic flux at the null, while for an X-point there is reconnection of magnetic flux. The singularity of $\mathbf{w}$ at magnetic $\mathrm{X}$-points in $2 \mathrm{D}$ is a signature of the fact that the reconnection process involves magnetic field lines being cut and rejoined at the X-point. In other words, the field line connectivity changes in a discontinuous manner at the null. Since $\mathbf{w}$ is smooth and continuous everywhere except at the X-point, field lines evolve as if they are reconnected at this point only. Hence, the reconnection of magnetic field lines occurs in a one-to-one pairwise fashion at a single point. This 2D reconnection scenario is demonstrated in Figure 2, where some representative flux tubes are plotted (from ideal comoving footpoints marked grey (green online) and black). The images in the figure show snapshots from an animation of flux tubes reconnecting in a kinematic steady-state solution with $\mathbf{B}=\left(y, k^{2} x, 0\right)$ and a localised diffusion region around the $\mathrm{X}$-point.

Perhaps surprisingly, it turns out that none of the above properties of $2 \mathrm{D}$ reconnection carry over into three dimensions. In general, in the presence of a localised non-ideal region (i.e. localised region within which $\mathbf{E} \cdot \mathbf{B} \neq 0$ ), a flux transporting velocity $\mathbf{w}$ does not exist anywhere in the vicinity of the diffusion region (for a proof, see Priest et al. (2003)). The result is that, if one follows magnetic field lines from footpoints comoving in the ideal flow, they appear to split as soon as they enter the non-ideal region, and their connectivity changes continually and continuously as they pass through the non-ideal region (see Figure 3). In other words, between any two neighbouring times $t$ and $t+\delta t$, every field line threading the non-ideal region experiences a change in connectivity. Consequently, magnetic field lines are not reconnected in a one-to-one fashion as in 2D. To illustrate, let us consider two field lines which are about to enter the diffusion region, one of which connects plasma elements labelled A and B, the other of which connects plasma elements labelled C and D (as in Figure 3). Then if the field lines are chosen such that after reconnection A connects to $\mathrm{C}$, then $\mathrm{B}$ will not be connected to D. This property has profound implications for the way in which the magnetic flux is restructured by the three-dimensional reconnection process - we can no longer think of a simple cut and paste of field line pairs.

The above properties are demonstrated in Figure 3. Representative flux tubes are traced from four cross sections, chosen such that at the initial time they form a pair a flux tubes. The plots are based on the steady-state kinematic solution of Hornig and Priest $(2003)$, with $\mathbf{B}=\left(y, k^{2} x, 1\right)$ and a diffusion region, $D$, localised around the origin. Note that in this solution the resistivity has been localised in order to obtain an analytical solution - however, the above-described topological properties of the flux evolution are not dependent on this localisation, and are still present when the non-ideal region is self-consistently localised through the formation of a localised current layer (as discussed later). As the flux tubes enter the diffusion region, they immediately begin to split, with field lines from cross-sections A and B (say) no longer being coincident. In frames 2-5 of the figure, the apparent 'flipping' (or 'slip-running') of field lines is demonstrated. The solid sections of the flux tubes are traced from ideal comoving footpoints (marked 

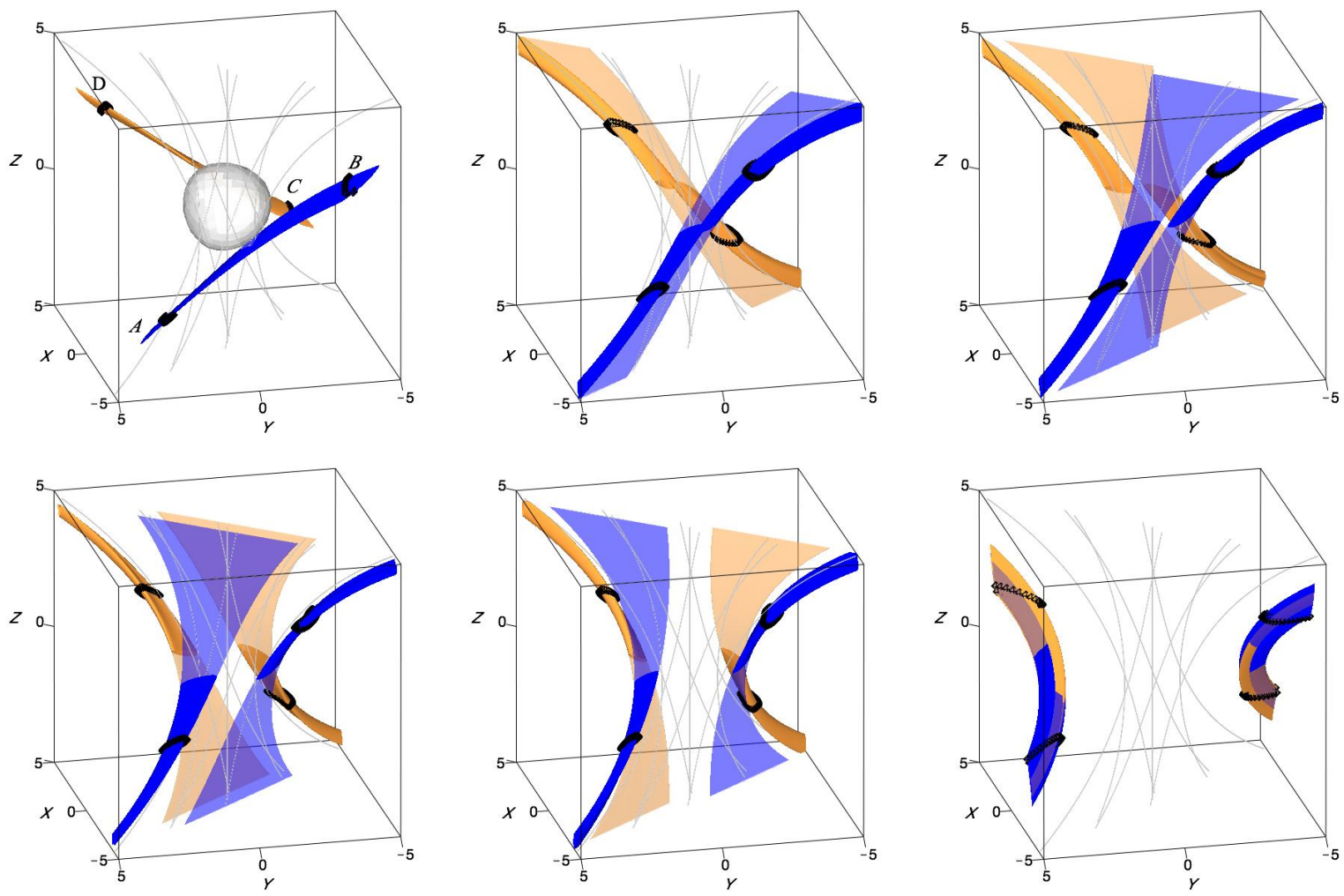

Figure 3: Reconnection of two representative flux tubes in the magnetic field $\mathbf{B}=\left(y, k^{2} x, 1\right)$, with $k=1.2$. The flux tubes are traced from ideal comoving footpoints (marked black), and the solid sections move at the local plasma velocity (outside $D$ ), while the transparent sections correspond to field lines that pass through the diffusion region. A localised diffusion region (shaded surface in the first frame) is present around the origin.

black) and move at the local plasma velocity (outside $D$ ), while the transparent sections correspond to field lines traced into and beyond the diffusion region, and appear to flip past one another at a velocity that is different from the local plasma velocity (until they exit the non-ideal region). Note that while in the initial state we began with two flux tubes, in contrast to the 2D case (shown in Figure 2), after reconnection (final frame, Figure 3) the four cross-sections do not match up to form two unique flux tubes.

The above 3D reconnection properties hold regardless of the topology of the magnetic field in the vicinity of the reconnection region: for further details, the reader is referred to the papers by Schindler et al. (1988); Hornig (2001, 2007a b); Priest et al. (2003). However, there are some profound differences that do occur when different magnetic field topologies are considered, so that as a result we may consider that there are a number of different magnetic reconnection regimes in $3 \mathrm{D}$. These regimes can be split into non-null reconnection (including reconnection in QSLs), null point reconnection (which has recently been categorised in a new way (Priest and Pontin, 2009) in response to new numerical experiments), and separator reconnection. In the following sections, we go on to consider each of these regimes in turn. 


\section{3D magnetic reconnection regimes: non-null reconnection}

As discussed above, in three dimensions, magnetic reconnection may occur in current layers which are not associated with magnetic nulls. The continuous change of connectivity of field lines traced from comoving footpoints has led to such reconnection being termed variously magnetic flipping (Priest and Forbes, 1992) or slip-running reconnection (if the virtual flipping velocity exceeds some threshold, Aulanier et al. (2006)). As discussed above, there are many different mechanisms by which the current layers may form. However, some basic properties of the resulting reconnection process will be universal.

A major step in understanding the properties of 3D non-null reconnection has been made by Hornig and Priest (2003). They considered the kinematic problem in which Ohm's law and Maxwell's equations are solved but the equation of motion is neglected (although it turns out that the solution solves the equation of motion in the limit of slow flows). The solution is obtained by imposing a steady-state magnetic field and plasma resistivity, and solving Ohm's law for the electric field and plasma velocity via

$$
\Phi=\int \eta \mathbf{J} \cdot \mathbf{B} d s, \quad \mathbf{E}=-\nabla \Phi, \quad \mathbf{v}_{\perp}=\frac{(\mathbf{E}-\eta \mathbf{J}) \times \mathbf{B}}{B^{2}},
$$

where $\mathbf{v}_{\perp}$ is the plasma velocity perpendicular to the magnetic field - the parallel component being arbitrary in this approximation. In order to obtain an analytical solution, the magnetic field and resistivity profiles must then be chosen in combination such that the first equation may be integrated.

Hornig and Priest (2003) chose a magnetic field consisting of a hyperbolic X-point plus a uniform field, specifically $\mathbf{B}=B_{0}\left(y, k^{2} x, 1\right) / L$. This linear magnetic field has the advantage that the field line mapping and its inverse can be expressed in closed form - a property that makes it possible to find closed-form solutions. Since the magnetic field is linear, the current within the volume is uniform: $\mathbf{J}=\left(0,0, B_{0}\left(k^{2}-1\right) / L \mu_{0}\right)$. Since the authors' aim was to study an isolated $3 \mathrm{D}$ reconnection process the generic case in astrophysical plasmas - the resistivity was chosen to be localised around the origin. The resulting electric and velocity fields are therefore fully $3 \mathrm{D}$, and magnetic field lines can be traced from ideal footpoints on either side of the diffusion region, since the product $\eta \mathbf{J}$ is localised.

Solving Equations (3), one can show that the plasma flow required to maintain this steady state configuration is a counter-rotational flow. More precisely, the flow is confined to field lines which thread the non-ideal region, with field lines above and below the non-ideal region (with respect to the direction of $\mathbf{B}$ ) rotating in opposite senses. One can demonstrate that this is a necessary property of the solution that follows directly from the presence of a 3D-localised parallel electric field within a region of nonvanishing magnetic field. That is, this property is independent of the specific choice of spatial profiles of $\mathbf{B}$ and $\eta$, or indeed the fact that $\eta$ rather than $\mathbf{J}$ is localised (a localisation of the current would be a more physically plausible way to localise $E_{\|}$, but is not compatible with the method of solution). This is clear if one considers the potential drop around the closed loop displayed in Figure 4, which must be zero since $\nabla \times \mathbf{E}=\mathbf{0}$. Now, $\mathbf{E} \cdot \mathbf{B} \neq 0$ along the central field line and since $\mathbf{E}$ does not change sign along that line then there must be a potential drop along $C 1$. Since $\mathbf{E} \cdot \mathbf{B}=0$ for any field line lying wholly outside the non-ideal region, there is no potential drop along $C 4$. It is therefore clear from the figure that it is necessary to have non-zero potential drops along the radial lines marked $C 2$ and $C 3$ (of opposite signs than on $C 1$ ). This implies a radial electric field component of opposite sign on $C 2$ and $C 3$. Since this particular choice of loop is not unique, we conclude that there must be an electric field component along any such radial line, and the combination of a radial electric field with a vertical component of the magnetic field implies that there must be an azimuthal flow within this envelope of flux that threads the non-ideal region.

As a consequence of the counter-rotational flows, field lines followed from the ideal region above and below the non-ideal region appear to undergo a 'rotational slippage' with respect to one another. This rotational slippage is quantified by the reconnection rate calculated in Equation (1). It is worth emphasising that this characteristic flow structure for 3D non-null reconnection is very different to the 


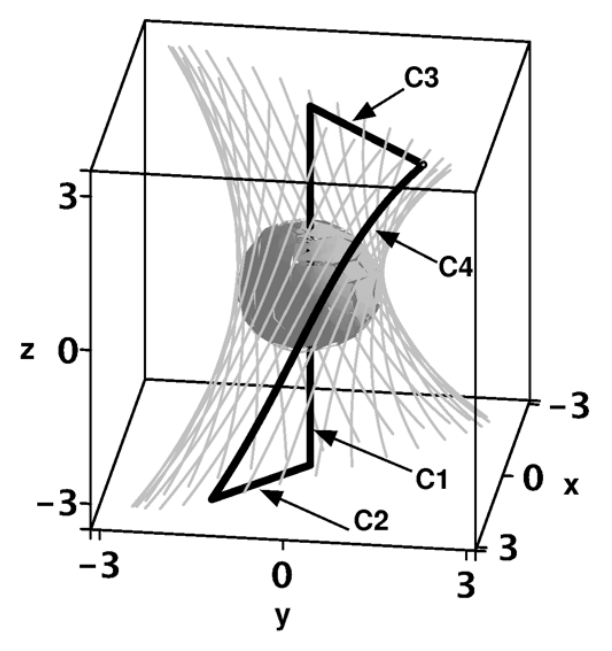

Figure 4: Potential drop around a closed circuit for the non-null reconnection solution of Hornig and Priest (2003).

classical 2D reconnection picture in which the characteristic flow structure is of stagnation type. The counter-rotational flows are a signature of the helicity production (decay) in 3D reconnection: if one writes down an evolution equation for the magnetic helicity then $\mathbf{E} \cdot \mathbf{B}$ appears as a source term. The solution of Hornig and Priest (2003) allows for the addition of an ideal flow via the constant function of integration in the integral in Equation (3). This function must be independent of $s$, i.e. constant along field lines, but may vary from one field line to another. The authors considered the effect of adding a flow with a hyperbolic structure in the $x y$-plane to transport magnetic flux into and out of the diffusion region (by adding a term proportional to $x_{0} y_{0}$ to $\Phi$, where $\left(x_{0}, y_{0}\right)$ is the point of intersection of a field line with the $z=0$ plane). The result is that field lines are brought into the non-ideal region, are split apart by the counter-rotational flows, and exit differently connected in opposite quadrants of the flow. The evolution of a particular pair of flux tubes for one of these solutions is shown in Figure 3 .

One should note that the idea of a localised region of non-zero $E_{\|}$being associated with a rotation in the plane perpendicular to the field has been described before by e.g. Hesse (1991). The above solution has been refined and put on a firm footing by solving the full system of MHD equations using an expansion scheme (Wilmot-Smith et al., 2006, 2009c; Al-Salti and Hornig, 2009), and the properties of the solution were also verified in a resistive MHD simulation (Pontin et al., 2005a). The solution describes the properties of a generic 3D reconnection process in a steady-state magnetic field in the absence of null points. In recent years various numerical simulations have explored the effects of $3 \mathrm{D}$ reconnection in more or less complicated magnetic field configurations. Notably, Linton et al. (2001) and Linton and Priest (2003) have investigated the interaction of magnetic flux tubes in an otherwise field-free environment, and discovered different possible interactions ('merge', 'bounce', 'tunnel' and 'slingshot') depending on the relative orientations of the tubes. In addition, recent simulations have shown that the local structure of the reconnection site during a non-null reconnection process need not necessarily be hyperbolic, but may also be elliptic (e.g. Wilmot-Smith et al., 2010). 


\section{3D magnetic reconnection regimes: null point reconnection}

\subsection{Kinematic models}

As discussed above, 3D magnetic null points have been proposed as possible sites of magnetic reconnection, due to the singularities that appear there in ideal kinematic models. The tendency of 3D null point structures to collapse (in the same way as $2 \mathrm{D}$ X-points) to generate currents locally has been studied by a number of authors (e.g. Klapper et al., 1996, Bulanov and Sakai, 1997; Mellor et al., 2003). Furthermore, investigations by Pontin and Craig (2005) suggest that current singularities are a natural consequence of an ideal MHD evolution in the vicinity of a line-tied 3D null.

Early models for 3D null point reconnection were proposed by Priest and Titov (1996) who considered the ideal kinematic limit and a current-free magnetic null. However, Pontin et al. (2004, 2005b) showed that the possible magnetic flux evolutions are very different when a localised diffusion region is included around the null point. They performed a similar kinematic analysis to that of Hornig and Priest (2003), again imposing a steady-state magnetic field and resistivity profile, and solving for the corresponding electric field and plasma flow. It was found that the nature of the magnetic reconnection is crucially dependent on the orientation of the electric current at the null point.

If the current is directed parallel to the spine of the null, then there are counter-rotational flows, centred on the spine (Pontin et al., 2004, Wyper and Jain, 2010). The change of connectivity that results from the reconnection process therefore takes the form of a rotational slippage similar to that discovered in the non-null case described above (see Figure 5). Importantly, there is no flux transport across either the spine or fan. In the simple model of Pontin et al. (2004) the reconnection rate is given by the integrated parallel electric field along the spine (by symmetry). The reconnection rate so determined quantifies the rotational slippage. Specifically, it measures the difference between the rate of (rotational) flux transport in the ideal region on either side of the diffusion region - i.e. the difference in the rate of flux transport through the two surfaces ('A' and 'B') shown in Figure 6.

By contrast, when the current is directed parallel to the fan surface (and is non-zero at the null itself), plasma flows cross both the spine and fan of the null, transporting flux both through/around the spine line, and across the fan separatrix surface (Pontin et al. 2005b), as shown in Figure 7. In this case, the reconnection rate can be shown to quantify the rate at which magnetic flux is transported across the separatrix surface in the ideal region - an interpretation that more closely resembles the two-dimensional picture.

The above described solutions suggest two main modes of magnetic reconnection at 3D nulls. However, in these kinematic models the diffusion region was artificially localised. The question still remains as to what types of current concentrations form self-consistently at 3D nulls in the dynamic regime. This has been investigated in a series of numerical simulations (Rickard and Titov, 1996, Galsgaard et al., 2003 Pontin and Galsgaard, 2007, Pontin et al., 2007a). The results have led Priest and Pontin (2009) to propose a new categorisation of $3 \mathrm{D}$ null point reconnection regimes, as follows.

\subsection{Torsional spine and fan reconnection}

Rickard and Titov (1996) and Pontin and Galsgaard (2007) investigated the propagation of disturbances towards symmetric 3D null points (where the fan eigenvalues are equal). In both studies, a general disturbance was decomposed into rotations (in planes perpendicular to the spine) and shearing motions. Rotational motions were found to behave in an essentially Alfvénic manner: they propagate along field lines, and accumulate around the spine line or fan plane. The locations of the spine and fan themselves remain undisturbed from their orthogonal potential configuration. These results are analogous to the properties of Alfvén wave propagation towards 2D X-points, summarised by McLaughlin et al. (2010). In each case, due to the hyperbolic geometry of the magnetic field, the current intensifies as the length 

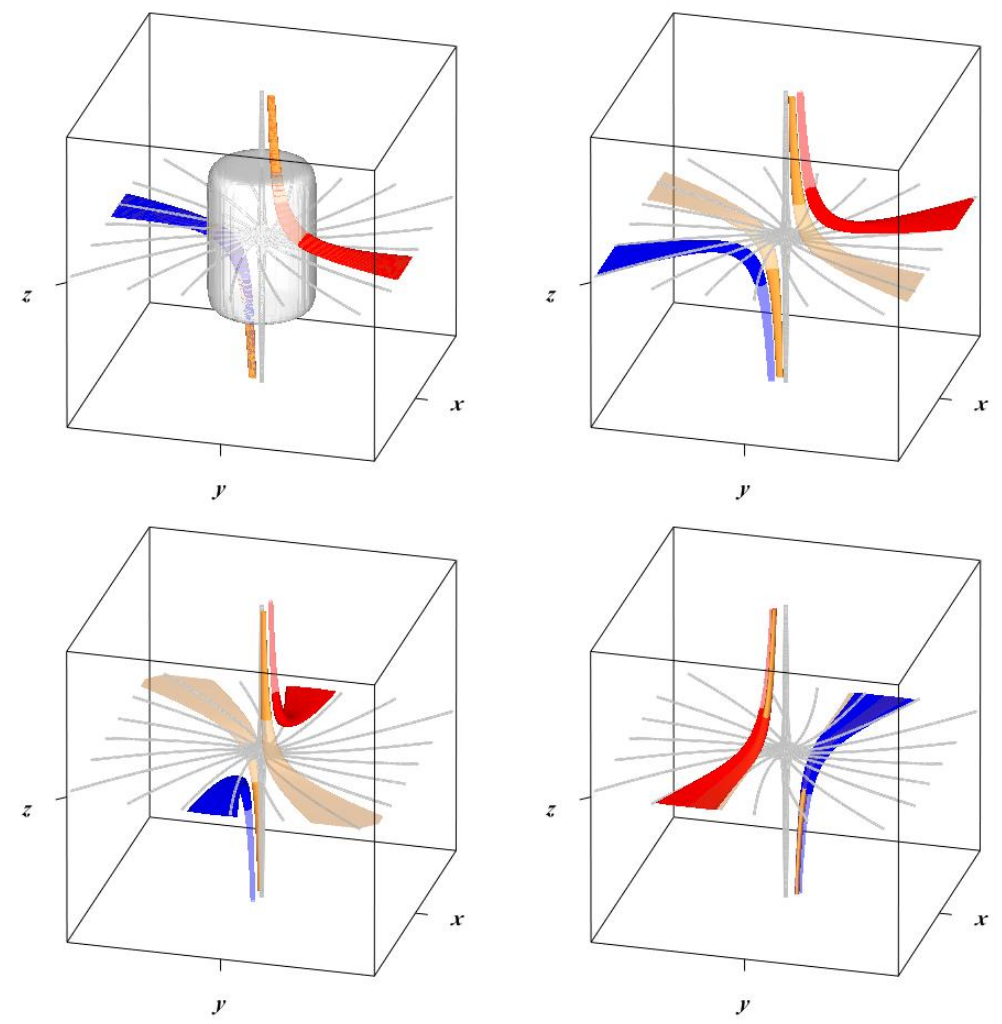

Figure 5: Reconnection of two representative flux tubes in the magnetic field $\mathbf{B}=(r, j r / 2,-2 z)$ in cylindrical polar coordinates, corresponding to current directed parallel to the spine (with $j=1$ ). A localised diffusion region is present around the null point, shown by the shaded surface in the first frame. Flux tubes are traced from four ideal comoving footpoints, with their extensions that pass through the diffusion region rendered as transparent, as in Figure 3.

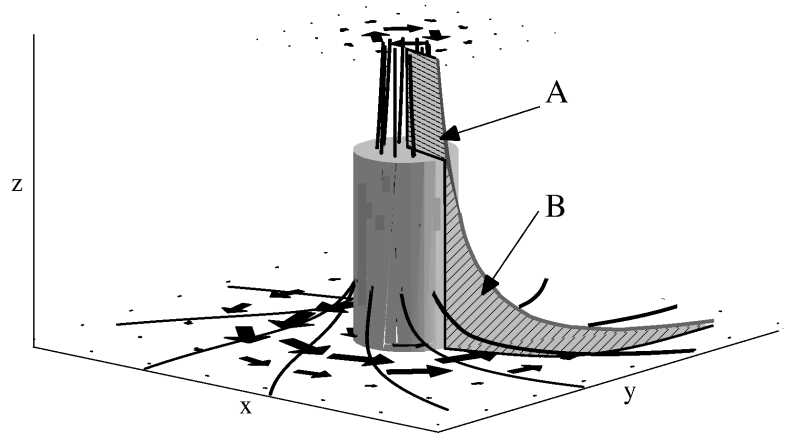

Figure 6: Interpretation of the reconnection rate for null point reconnection with a spatially-uniform spine-aligned current (kinematic solution with $\mathbf{B}=(r, j r / 2,-2 z)$ in polar coordinates, $j=1$ ). Heavy black lines are magnetic field lines, the grey cylinder represents the diffusion region, and the arrows show the plasma flow in two representative planes of $z=$ const. Magnetic flux is transported through surfaces ' $\mathrm{A}$ ' and 'B' at different rates. 

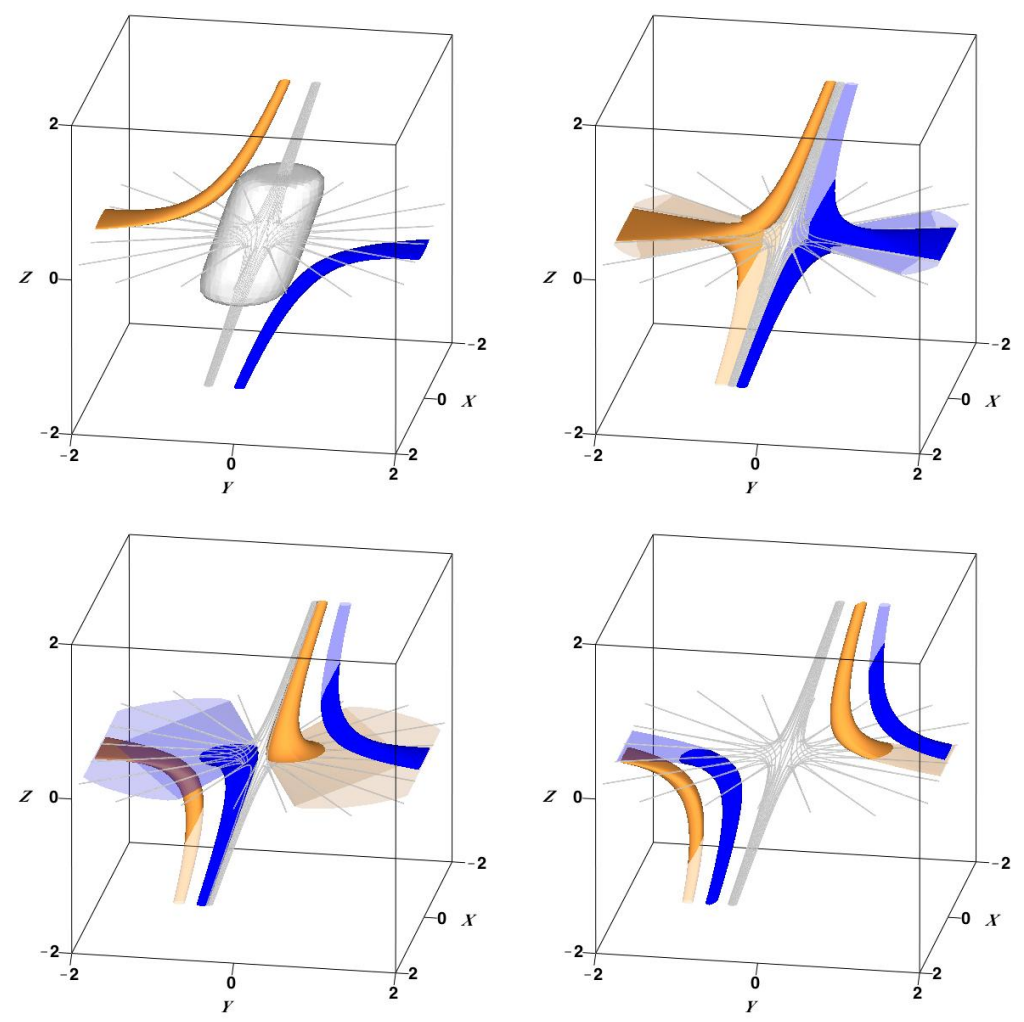

Figure 7: Reconnection of two representative flux tubes in the magnetic field $\mathbf{B}=(x, y-j z,-2 z)$, corresponding to current directed parallel to the fan plane (with $j=1$ ). A localised diffusion region is present around the null point, shown by the shaded surface in the first frame. Flux tubes are traced from four ideal comoving footpoints, with their extensions that pass through the diffusion region rendered as transparent, as in Figure 3 . 


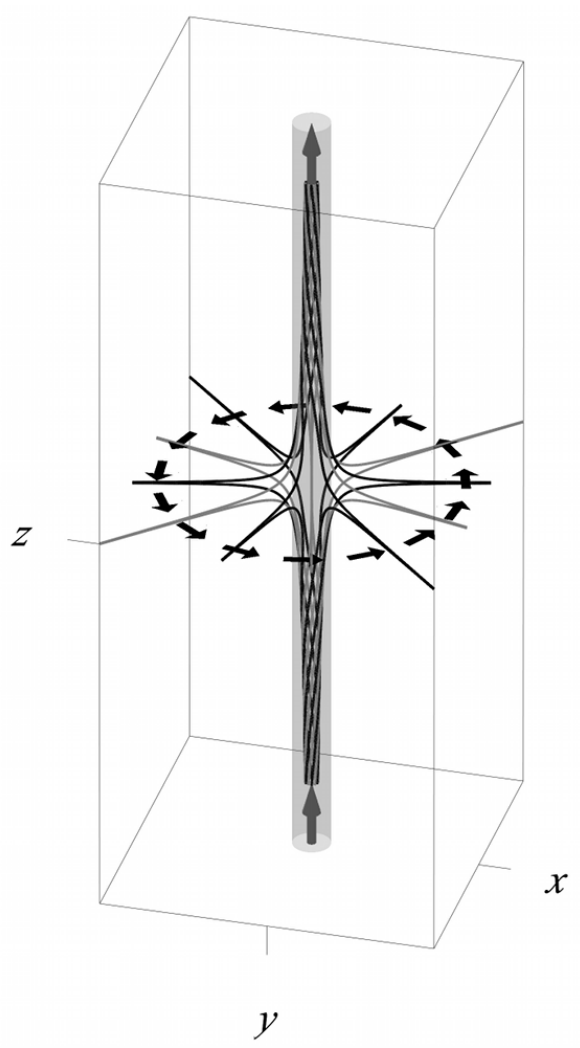

Figure 8: Schematic diagram of torsional spine reconnection at an isolated null. Black and grey lines are magnetic field lines, the shaded surface is a current density isosurface, the grey arrows indicate the direction of the current flow, while the black arrows indicate the driving plasma velocity.

scales perpendicular to the spine or fan become shorter. This intensification ceases once these length scales become sufficiently short that diffusion becomes important.

Torsional spine reconnection occurs in response to a rotational disturbance of the fan plane. The disturbance propagates to the spine, around which an extended tube of current forms. This current tube is generated by a twisting of the magnetic field lines locally around the spine line, and as such the current vector is directed parallel to the axis of the tube, i.e. parallel to the spine, see Figure 8. Due to the orientation of the current, the magnetic reconnection that occurs within the current layer takes the form of a rotational slippage, as discussed above. In a continuously driven system, a quasi-steady state will be reached when the rotational advection that increases the twist of the magnetic field around the spine and thus the current - is balanced by this rotational slippage.

Torsional fan reconnection occurs in response to a rotational disturbance around the spine. The perturbation propagates as a helical Alfvén wave towards the fan, where a planar current layer develops (Galsgaard et al., 2003), as shown in Figure 9. While away from the null the current is dominated by its components parallel to this plane, it flows through the null parallel to the spine. Therefore a rotational slippage of magnetic flux is expected from the kinematic models, and indeed this is the form that the reconnection takes. Again, a quasi-steady current layer would be expected to form once the increased twisting of field lines is balanced by this rotational slippage. 


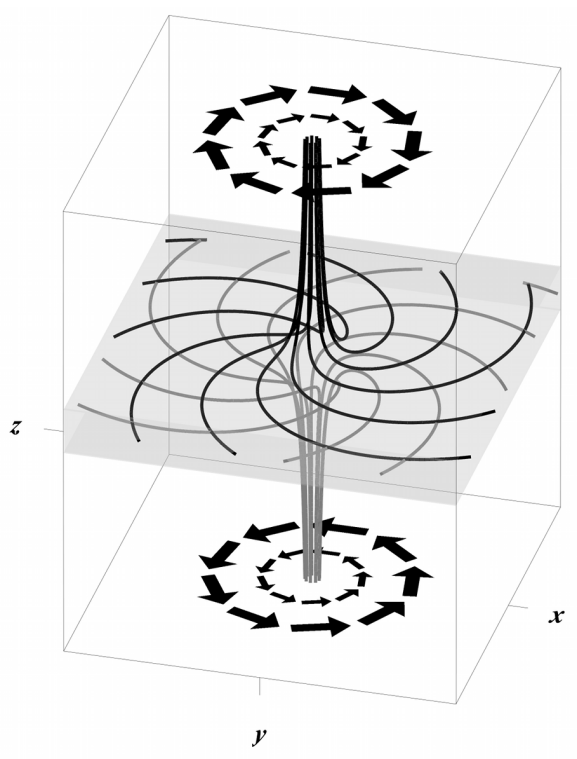

Figure 9: Schematic diagram of torsional fan reconnection at an isolated null. Black and grey lines are magnetic field lines, the shaded surface is a current density isosurface, and the black arrows indicate the driving plasma velocity.

\subsection{Spine-fan reconnection}

The torsional spine and torsional fan reconnection modes discussed above require a rather organised rotational driving motion - and it is thus anticipated that the most common regime of reconnection to occur at 3D nulls is the spine-fan reconnection mode. This mode of reconnection occurs within a current sheet that is localised in all three dimensions around the null. Such a current concentration is found to form when a shear disturbance of either the spine or the fan occurs (Pontin et al., 2007a). Since in this case the disturbance propagates across magnetic field lines as it localises at the null, its behaviour has the properties of a magnetoacoustic wave. The current layer at the null is formed by a local collapse of the magnetic field - the spine and fan collapse towards one another, with the current sheet locally spanning them both, as depicted in Figure 10. The plane in which the spine and fan collapse is selected by the plane of the shear disturbance, and in this plane the spine, fan, and current layer together form a Y-type structure. The current flows through the null perpendicular to this shear plane, and thus parallel to the fan surface. As the null point collapses, magnetic flux is transported through both the spine and the fan, as predicted by the kinematic model due to the current orientation. Although the majority of previous studies considered the perturbation of a symmetric null point, Al-Hachami and Pontin (2010) have demonstrated that when a generic non-symmetric null is considered, while the qualitative properties of the reconnection process are preserved, the dimensions of the diffusion region and reconnection rate can vary strongly.

It is worth noting the relation of the spine-fan reconnection regime to the steady-state mathematical models proposed by Craig et al. (1995); Craig and Fabling (1996). These are exact solutions of the incompressible MHD equations, and are often termed 'reconnective annihilation' models, since they involve current layers that extend to infinity along either the spine or the fan. The solutions are constructed by super-imposing 1D or 2D disturbances consisting of infinite, straight field onto a background potential null. The solutions involving a planar current layer in the fan have been demonstrated to be dynamically accessible (Craig and Fabling, 1998). However, in order to maintain the planar nature of the current layer (which is imposed by the restrictive but necessary choice of low-dimensionality disturbance fields), 


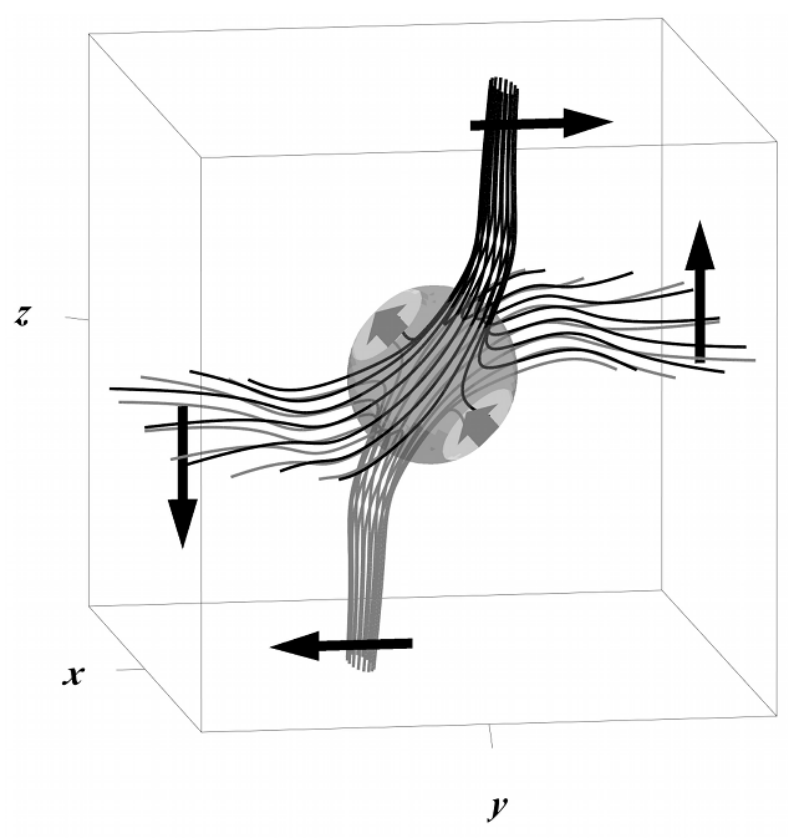

Figure 10: Schematic diagram of spine-fan reconnection at an isolated null. Black and grey lines are magnetic field lines, the shaded surface is a current density isosurface, the grey arrows indicate the direction of the current flow, while the black arrows indicate the driving plasma velocity.

a large pressure gradient is required within the current sheet. It has been shown in MHD simulations that when the incompressibility condition is relaxed, the pressure gradient is not able to balance the Lorentz force acting within the current layer, whereupon the magnetic field collapses to form a localised current layer at the null as described above (Pontin et al. 2007b). On the other hand, indications are that the spine reconnective annihilation models are not dynamically accessible (Titov et al., 2004, Pontin et al. 2007b).

\section{3D magnetic reconnection regimes: separator reconnection}

In addition to reconnection at isolated 3D null points and in their absence, reconnection may also occur in a configuration containing multiple magnetic nulls connected by one or more separator field lines. The form of current layers at such separator field lines has been investigated by Longcope and Cowley (1996), while Longcope (1996) has proposed that currents will naturally focus at separator lines during relaxation processes in the solar corona. Early kinematic models in current-free magnetic fields predicted that separator reconnection would involve a simple cut-and-paste of field line pairs at the separator line (Lau and Finn, 1990; Priest and Titov, 1996). In the absence of any current, the magnetic field in a plane orthogonal to the separator has a perpendicular X-type structure, and the reconnection was envisaged projected on such a 2D plane - as being much like 2D X-point reconnection, as depicted in Figure 11. Such a picture was qualitatively backed up in numerical simulations by Galsgaard et al. (2000). However, new results throw serious doubts on these simplified pictures.

Separator reconnection is perhaps the least well-understood 3D reconnection regime. One major unknown property is the typical distribution of the current along the separator, and therefore whether separator reconnection shares more in common with the null or non-null reconnection regimes. Reconnective annihilation models for incompressible plasmas suggest that reconnection may be focussed either at the nulls or between them along the separator (Craig et al., 1999, Pontin and Craig, 2006). One 

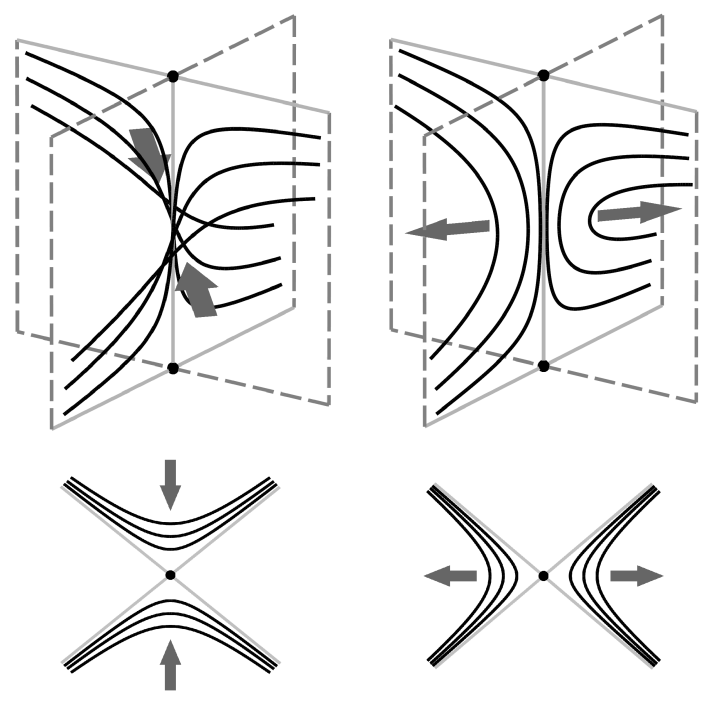

Figure 11: Cartoon showing field lines before (left) and after (right) reconnection at a separator in the simplified kinematic picture. Black circles mark the nulls and arrows indicate the direction of plasma / field line motion. Lower images show the view looking along the direction of the separator from above.

thing that is clear is that the picture of cut-and-paste one-to-one rejoining of field lines at the separator line is over-simplified. When a localised current layer forms around a separator, the reconnection within the associated diffusion region must conform to the properties described in Section 3 . Therefore there will be a continuous reconnection of field lines within the volume surrounding the separator. Indeed, as demonstrated by Parnell et al. (2010), the structure of the magnetic field in the vicinity of the diffusion region may be significantly more complex than previously expected. The authors investigated in detail the evolution of the magnetic field in a simulation in which two patches of opposite polarity magnetic flux on the boundary were driven past one another (see also Haynes et al., 2007). While there were only two isolated, unconnected nulls in the initial configuration, during the evolution a number of separators were formed, around which the current was found to be focussed. Although a separator must have a hyperbolic field (in the plane locally perpendicular to the field) near the nulls, it can have hyperbolic as well as elliptic structure away from the nulls. In the simulation, changes of the structure both along the separator as well as in time were found. It is natural that an elliptic field such as this be present in the vicinity of a strong current along the separator. This is demonstrated in Figure 12, Field lines are plotted for a magnetic field consisting of a potential component defining a separator structure plus a component that defines a line current. Specifically, the magnetic field is

$$
\begin{aligned}
\mathbf{B}= & \left(x\left(z-3 z_{0}\right), y\left(z+3 z_{0}\right), z_{0}^{2}-z^{2}+\frac{1}{2}\left(x^{2}+y^{2}\right)\right) \\
& +j\left(-40 y e^{-20 x^{2}-20 y^{2}}, 40 x e^{-20 x^{2}-20 y^{2}}, 0\right)
\end{aligned}
$$

which contains nulls at $x=y=0, z= \pm z_{0}$, with a separator located at $-z_{0}<z<z_{0}$. Part (a) shows the case of a relatively weak current, $j=0.1$, for which the magnetic field around the separator is hyperbolic. However, when the current is increased $(j=1$, shown in part (b)), the field lines spiral around the separator. Clearly, reconnection at a separator with elliptic local magnetic field must be rather different to the simple early models. Indeed, Parnell et al. (2010) identified the presence of counter-rotating flows around the separator on either side of localised enhancements in the parallel electric field, a signature of the non-null reconnection described in Section 4. 

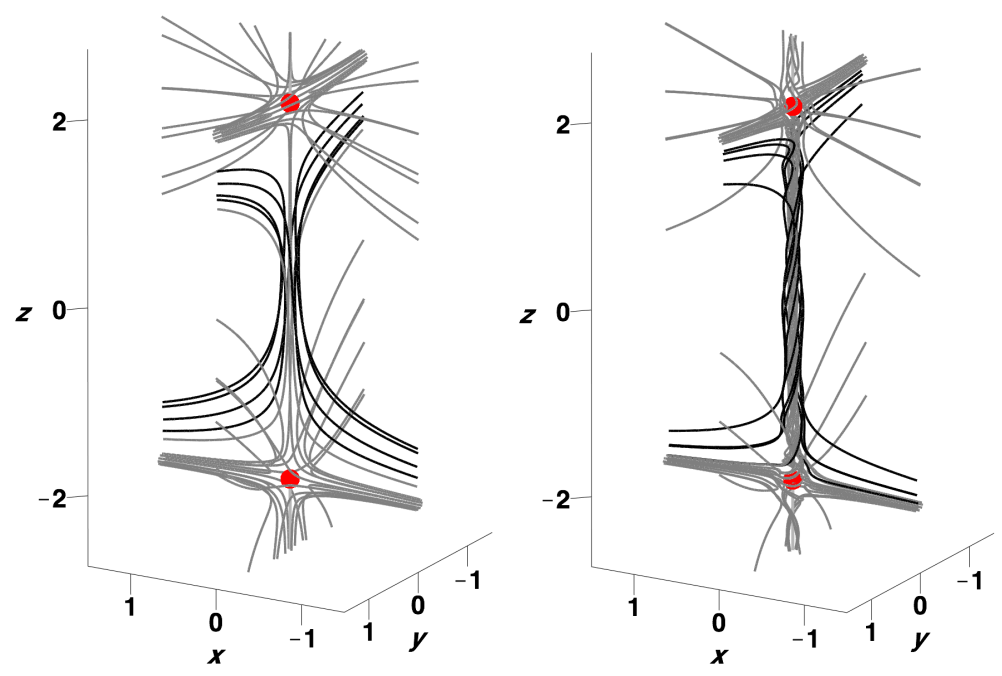

Figure 12: Field lines for the magnetic field defined by Equation (4), with $z_{0}=2$ and (a) $j=0.1$ and (b) $j=0.4$. The separator runs along the $z$-axis between the two disks which represent the nulls (red online). Grey field lines are traced from footpoints near the spines of the nulls, while black field lines are traced from a circle around the separator, centred at the origin in the $z=0$ plane.

\section{3D magnetic reconnection in simulations and observations}

Magnetic reconnection is a key ingredient for many astrophysical processes, from the collapse of accretion disks through to stellar dynamos and flares. Identification of the 3D magnetic reconnection regimes described above has assisted with the diagnosis of results from - and been motivated by - observations and numerical simulations. Those observations and simulations that invoke specific 3D reconnection models tend to come from the study of the Solar atmosphere and Earth's magnetosphere, as comparatively well-resolved data is available for these environments.

$3 \mathrm{D}$ reconnection in the absence of null points is seen, for example, in 3D simulations of the Parker coronal heating scenario (e.g. Hendrix and van Hoven, 1996, Galsgaard and Nordlund, 1996; Rappazzo et al. 2008). While the topology of the magnetic field in the vicinity of the reconnection sites is not determined in these studies, the presence of a strong background 'guide' field ensures the absence of topological features within the domain. In the solar atmosphere, observed sites of energy release are often taken as signatures of a local magnetic reconnection process. While the magnetic field in the corona cannot be directly measured, many studies have attempted to determine the local magnetic field structure around the reconnection site by extrapolation from vector magnetograms. A number of these studies find the locations of energy release to be well-correlated with the locations of quasi-separatrix layers within the coronal volume (e.g. Démoulin et al., 1994; Démoulin et al., 1997; Mandrini et al., 2006; Titov et al., 2008). Furthermore, numerical modelling of the loss of stability and subsequent eruption of flux ropes has implicated reconnection in a QSL beneath the flux rope (Titov and Démoulin, 1999; Kliem et al. 2004, Titov et al., 2008). A new technique for quantifying the reconnection rate in such simulations using so-called 'slip-squashing factors' has recently been proposed (Titov et al., 2009). Observations of the solar corona give strong indications that the continuous change of connectivity associated with 3D reconnection truly occurs there. Aulanier et al. (2007) reported observations by the X-ray telescope onboard the Hinode satellite of slippage of coronal loops which implicated non-null (slip-running) reconnection in a QSL. Further evidence was presented by Masson et al. (2009) who described TRACE (Transition Region And Coronal Explorer) observations of propagating bright sources along a flare ribbon associated with 
the fan surface of a coronal null point, related to the flipping of field lines during spine-fan reconnection at the null.

Extrapolations of magnetic fields in solar active regions indicate that different magnetic reconnection modes are important for different flare events. In particular, while the references above found no 3D magnetic nulls in the vicinity of energy release events, there are also a number of observations which suggest that null points are a common feature in flaring or eruptive locations in active regions and the quiet sun (e.g. Fletcher et al., 2001; Mandrini et al., 2006; Luoni et al., 2007; Ugarte-Urra et al., 2007; Török et al., 2009; Masson et al., 2009). Furthermore, while nulls are by no means found to be associated with all solar eruptions, a statistical study by Barnes (2007) showed that active regions containing nulls are more susceptible to yield eruptions. 3D null point reconnection, via the spine-fan reconnection mode, has been proposed as a mechanism for polar jets (Pariat et al., 2009) as well as for CMEs via the 'magnetic breakout' model (Antiochos et al., 1999, Lynch et al., 2008).

The hypothesis that current accumulates naturally at separator lines has been backed up by various numerical simulations (Galsgaard and Nordlund, 1997; Galsgaard et al., 1997; Haynes et al., 2007). Longcope et al. (2005) have inferred the presence of separator reconnection in the corona based on observations of the emergence of a new active region in the vicinity of a pre-existing active region. One of the great difficulties in diagnosing the reconnection mechanism in $3 \mathrm{D}$ numerical simulations has been to determine the topology of the magnetic field in the vicinity of the reconnection site. However, great progress has recently been made with the development of new algorithms to effectively determine the topological 'skeleton' of complex 3D magnetic fields (Haynes and Parnell, 2007, 2010). While many observational signatures of reconnection in the Earth's magnetosphere have been treated as quasi-2D and interpreted as such, it is now clear that 3D topology is also of crucial importance there. Examining the magnetic topology of global magnetospheric simulations (with Northward IMF, clock angle $45^{\circ}$ ), Dorelli et al. (2007) discovered clusters of nulls in the cusp regions connected on the dayside by a separator line. Furthermore, the presence of multiple 3D magnetic nulls in the tail current sheet connected by webs of separators has been inferred from in-situ observations made by the Cluster spacecraft (Xiao et al., 2007, Deng et al., 2009), suggesting that some kind of complex 3D tearing-like process may be occurring there.

\section{Summary and outlook}

Magnetic reconnection is a universal process in astrophysical plasmas. It facilitates the release of stored magnetic energy by permitting changes of magnetic topology (or just changes of field line connectivity for reconnection in the absence of topological structures) and as such is a key ingredient of many energetic processes in these environments. It is only in recent years that the rich geometrical and topological structure of these astrophysical plasmas has begun to be appreciated, following great advances in observations.

In $3 \mathrm{D}$, the qualitative properties of reconnection are much richer than in $2 \mathrm{D}$, where reconnection occurs only at magnetic X-type null points. There are many topological and geometrical features of a magnetic field that may be favourable sites for current growth. 3D reconnection processes in different magnetic field structures have different characteristic properties, and as such can be classified into separate regimes. Reconnection may occur in the absence of any null points, with the presence of counter-rotational flows on either side of the diffusion region being a characteristic feature. Reconnection may also occur at isolated null points, with 'torsional spine', 'torsional fan' and 'spine-fan' reconnection modes thus far identified. The most common of these - spine-fan reconnection - involves a local collapse of the null to form a current sheet (focussed at the null) that locally spans both the spine and fan, with flux transfer through spine line and fan (separatrix) surface. Furthermore, reconnection may occur at separator lines connecting pairs of nulls, in which the properties of the reconnection process may share some properties with null or non-null reconnection modes. While each of the reconnection modes has its own characteristics, they all share 
some fundamental properties that make them distinct to $2 \mathrm{D}(\mathbf{E} \cdot \mathbf{B}=0)$ reconnection. In particular, the non-existence of a unique flux-conserving velocity anywhere within the diffusion region (i.e. for any field lines threading the diffusion region), which implies that reconnection occurs throughout the diffusion region, not at a single point as in $2 \mathrm{D}$. As a result, there is no one-to-one cut-and-paste rejoining of field lines.

While we are now beginning to understand some of the properties of $3 \mathrm{D}$ reconnection, there is much left to discover. Future advances are likely to be led by large-scale MHD simulations being developed alongside fundamental theory. Many important open questions remain, such as:

- What are the quantitative properties of the different 3D reconnection regimes? Thus far the vast majority of our knowledge is of a qualitative nature, and quantitative studies are required to probe, for example, the diffusion region dimensions. What is the range of possible reconnection rates in each regime, and what determines the reconnection rate? How do these numbers scale with different plasma parameters? While 3D reconnection studies have so far (by necessity) used the MHD approximation, it will be important in the future to investigate the implications of including additional physics such as Hall and electron pressure tensor electric fields in Ohm's law, to better model the physics within the diffusion region.

- What is the relationship between null, non-null, and separator reconnection?

- Which of these modes of reconnection is most important in more realistic (complex) magnetic fields - when the local models discussed above are embedded within global magnetic field configurations? One step towards answering this question would be to determine the observational signatures of the different reconnection regimes.

- What are the most common mechanisms of current sheet formation in complex 3D magnetic fields? How does the reconnection influence the global evolution of the magnetic field? (This may be more complex than previously appreciated - recent MHD simulations have shown that the distribution of reconnection sites may be highly complex and flux may be reconnected multiple times in generic 3D MHD evolutions Parnell et al. (2008); Pontin et al. (2011))

- How is the magnetic energy transferred to other forms, and ultimately dissipated, in 3D reconnection? This includes the important question of the pattern and efficiency of particle acceleration in each $3 \mathrm{D}$ reconnection regime.

\section{Acknowledgements}

This review is based on a paper presented at the 38th COSPAR assembly. The author would like to thank the organisers for the invitation to present the review, and gratefully acknowledges financial support from COSPAR. Thanks are also extended to Gunnar Hornig, Antonia Wilmot-Smith, Eric Priest, Clare Parnell and Klaus Galsgaard for ongoing fruitful discussions and collaborations, as well as comments that helped to improve this manuscript.

\section{References}

Al-Hachami, A.K., Pontin, D.I., 2010. Magnetic reconnection at 3D null points: Effect of magnetic field asymmetry. Astron. Astrophys. 512, A84.

Al-Salti, N., Hornig, G., 2009. On the solutions of three-dimensional non-null magnetic reconnection. Phys. Plasmas 16, 082101. 
Antiochos, S.K., DeVore, C.R., Klimchuk, J.A., 1999. A model for solar coronal mass ejections. Astrophys. J. 510, 485-493.

Aulanier, G., Golub, L., DeLuca, E.E., et al., 2007. Slipping magnetic reconnection in coronal loops. Science 318, 1588.

Aulanier, G., Pariat, E., Démoulin, P., Devore, C.R., 2006. Slip-running reconnection in quasi-separatrix layers. Solar Phys. 238, 347-376.

Barnes, G., 2007. On the relationship between coronal magnetic null points and solar eruptive events. Astrophys. J. Lett. 670, L53-L56.

Biskamp, D., 2000. Magnetic reconnection in plasmas. Cambridge University Press.

Browning, P.K., Gerrard, C., Hood, A.W., Kevis, R., van der Linden, R.A.M., 2008. Heating the corona by nanoflares: simulations of energy release triggered by a kink instability. Astron. Astrophys. 485, $837-848$.

Bulanov, S.V., Sakai, J., 1997. Magnetic collapse in incompressible plasma flows. J. Phys. Soc. Jpn. 66, $3477-3483$.

Craig, I.J.D., Fabling, R.B., 1996. Exact solutions for steady-state, spine, and fan magnetic reconnection. Astrophys. J. 462, 969-976.

Craig, I.J.D., Fabling, R.B., 1998. Dynamic magnetic reconnection in three space dimensions: Fan current solutions. Phys. Plasmas 5, 635-644.

Craig, I.J.D., Fabling, R.B., Heerikhuisen, J., Watson, P.G., 1999. Magnetic reconnection solutions in the presence of multiple nulls. Astrophys. J. 523, 838-848.

Craig, I.J.D., Fabling, R.B., Henton, S.M., Rickard, G.J., 1995. An exact solution for steady state magnetic reconnection in three dimensions. Astrophys. J. Lett. 455, L197-L199.

Démoulin, P., 2006. Extending the concept of separatrices to qsls for magnetic reconnection. Adv. Space Res. 37, 1269-1282.

Démoulin, P., Hénoux, J.C., Mandrini, C.H., 1994. Are null magnetic points important in solar flares? Astron. Astrophys. 285, 1023.

Démoulin, P., Bagala, L.G., Mandrini, C.H., Hénoux, J.C., Rovira, M.G., 1997. Quasi-separatrix layers in solar flares. II. Observed magnetic configurations. Astron. Astrophys. 325, 305-317.

Deng, X.H., Zhou, M., Li, S.Y., et al., 2009. Dynamics and waves near multiple magnetic null points in reconnection diffusion region. J. Geophys. Res. 114, A07216.

Dorelli, J.C., Bhattacharjee, A., Raeder, J., 2007. Separator reconnection at earth's dayside magnetopause under generic northward interplanetary magnetic field conditions. J. Geophys. Res. 112, A02202.

Fletcher, L., Metcalf, T.R., Alexander, D., Brown, D.S., Ryder, L.A., 2001. Evidence for the flare trigger site and three-dimensional reconnection in multiwavelength observations of a solar flare. Astrophys. J. $554,451-463$.

Fukao, S., Ugai, M., Tsuda, T., 1975. Topological study of magnetic field near a neutral point. Rep. Ion. Sp. Res. Japan 29, 133-139. 
Galsgaard, K., Nordlund, A., 1996. Heating and activity of the solar corona: 1. Boundary shearing of an initially homogeneous magnetic field. J. Geophys. Res. 101, 13445-13460.

Galsgaard, K., Nordlund, A., 1997. Heating and activity of the solar corona: 3. Dynamics of a low beta plasma with 3D null points. J. Geophys. Res. 102, 231-248.

Galsgaard, K., Priest, E.R., Nordlund, A., 2000. Three-dimensional separator reconnection - how does it occur? Solar Phys. 193, 1-16.

Galsgaard, K., Reddy, R.V., Rickard, G.J., 1997. Energy release sites in magnetic fields containing single or multiple nulls. Solar Phys. 176, 299-325.

Galsgaard, K., Titov, V.S., Neukirch, T., 2003. Magnetic pinching of hyperbolic flux tubes. ii. dynamic numerical model. Astrophys. J. 595, 506-516.

Haynes, A.L., Parnell, C.E., 2007. A trilinear method for finding null points in a three-dimensional vector space. Physics of Plasmas 14, 082107.

Haynes, A.L., Parnell, C.E., 2010. A method for finding three-dimensional magnetic skeletons. Physics of Plasmas 17, 092903.

Haynes, A.L., Parnell, C.E., Galsgaard, K., Priest, E.R., 2007. Magnetohydrodynamic evolution of magnetic skeletons. Royal Society of London Proceedings Series A 463, 1097-1115.

Hendrix, D., van Hoven, G., 1996. Magnetohydrodynamic turbulence and implications for solar coronal heating. Astrophys. J. 467, 887-893.

Hesse, M., 1991. Advances in solar system magnetohydrodynamics. Cambridge University Press: Cambridge. p. 221.

Hesse, M., Schindler, K., 1988. A theoretical foundation of general magnetic reconnection. J. Geophys. Res. 93, 5558-5567.

Hood, A.W., Browning, P.K., van der Linden, R.A.M., 2009. Coronal heating by magnetic reconnection in loops with zero net current. Astron. Astrophys. 506, 913-925.

Hornig, G., 2001. The geometry of reconnection, in: Ricca, R.L. (Ed.), An introduction to the geometry and topology of fluid flows. Kluwer, Dordrecht. pp. 295-313.

Hornig, G., 2007a. Fundamental concepts, in: Birn, J. and Priest, E.R. (Eds.), Reconnection of magnetic fields. Cambridge University Press. pp. 25-45.

Hornig, G., 2007b. 3D reconnection without nulls, in: Birn, J. and Priest, E.R. (Eds.), Reconnection of magnetic fields. Cambridge University Press. pp. 45-62.

Hornig, G., Priest, E.R., 2003. Evolution of magnetic flux in an isolated reconnection process. Phys. Plasmas 10, 2712-1721.

Hornig, G., Schindler, K., 1996. Magnetic topology and the problem of its invariant definition. Phys. Plasmas 3, 781-791.

Klapper, I., Rado, A., Tabor, M., 1996. A Lagrangian study of dynamics and singularity formation at magnetic null points in ideal three-dimensional magnetohydrodynamics. Phys. Plasmas 3, 4281-4283.

Kliem, B., Titov, V.S., Török, T., 2004. Formation of current sheets and sigmoidal structure by the kink instability of a magnetic loop. Astron. Astrophys. 413, L23-L26. 
Lau, Y.T., Finn, J.M., 1990. Three dimensional kinematic reconnection in the presence of field nulls and closed field lines. Astrophys. J. 350, 672-691.

Linton, M., Dahlburg, R.B., Antiochos, S.K., 2001. Reconnection of twisted flux tubes as a function of contact angle. Astrophys. J. 553, 905-921.

Linton, M., Priest, E.R., 2003. Three-dimensional reconnection of untwisted flux tubes. Astrophys. J. 595, 1259-1276.

Longcope, D.W., 1996. Topology and current ribbons: A model for current, reconnection and flaring in a complex, evolving corona. Solar Phys. 169, 91-121.

Longcope, D.W., Cowley, S.C., 1996. Current sheet formation along three-dimensional magnetic separators. Phys. Plasmas 3, 2885-2897.

Longcope, D.W., McKenzie, D., Cirtain, J., Scott, J., 2005. Observations of separator reconnection to an emerging active region. Astrophys. J. 630, 569.

Longcope, D.W., Parnell, C.E., 2009. The number of magnetic null points in the quiet sun corona. Solar Phys. 254, 51-75.

Longcope, D.W., Strauss, H.R., 1994. The form of ideal current layers in line-tied magnetic fields. Astrophys. J. 437, 851-859.

Luoni, M.L., Mandrini, H.H., Cristiani, G.D., Démoulin, P., 2007. The magnetic field topology associated with two M-flares. Adv. Space Res. 39, 1382-1388.

Lynch, G.J., Antiochos, S.K., DeVore, C.R., Luhmann, J.G., Zurbuchen, T.H., 2008. Topological evolution of a fast magnetic breakout CME in three dimensions. Astrophys. J. 683, 1192-1206.

Mandrini, C.H., Démoulin, P., Schmieder, B., Deluca, E.E., Pariat, E., Uddin, W., 2006. Companion event and precursor of the X17 flare on 28 October 2003. Solar Phys. 238, 293-312.

Masson, S., Pariat, E., Aulanier, G., Schrijver, C.J., 2009. The nature of flare ribbons in coronal null-point topology. Astrophys. J. 700, 559-578.

McLaughlin, J.A., Hood, A.W., de Moortel, I., 2010. Review article: MHD wave propagation near coronal null points of magnetic fields. Space Sci. Rev. , 62.

Mellor, C., Titov, V.S., Priest, E.R., 2003. Linear collapse of spatially linear 3D potential null points. Geophys. Astrophys. Fluid Dynamics 97, 489-505.

Ng, C.S., Bhattacharjee, A., 1998. Nonequilibrium and current sheet formation in line-tied magnetic fields. Phys. Plasmas 5, 4028-4040.

Pariat, E., Antiochos, S.K., DeVore, C.R., 2009. A model for solar polar jets. Astrophys. J. 691, 61-74.

Parker, E.N., 1972. Topological dissipation and the small-scale fields in turbulent gases. Astrophys. J. 174, 499.

Parnell, C.E., Haynes, A.L., Galsgaard, K., 2008. Recursive reconnection and magnetic skeletons. Astrophys. J. 675, 1656-1667.

Parnell, C.E., Haynes, A.L., Galsgaard, K., 2010. Structure of magnetic separators and separator reconnection. J. Geophys. Res. 115, A02102. 
Parnell, C.E., Smith, J.M., Neukirch, T., Priest, E.R., 1996. The structure of three-dimensional magnetic neutral points. Phys. Plasmas 3, 759-770.

Pontin, D.I., Bhattacharjee, A., Galsgaard, K., 2007a. Current sheet formation and non-ideal behaviour at three-dimensional magnetic null points. Phys. Plasmas 14, 052106.

Pontin, D.I., Bhattacharjee, A., Galsgaard, K., 2007b. Current sheets at three-dimensional magnetic null points: Effect of compressibility. Phys. Plasmas 14, 052109.

Pontin, D.I., Craig, I.J.D., 2005. Current singularities at finitely compressible three-dimensional magnetic null points. Phys. Plasmas 12, 072112.

Pontin, D.I., Craig, I.J.D., 2006. Dynamic 3D reconnection in a separator geometry with two null points. Astrophys. J. 642, 568-578.

Pontin, D.I., Galsgaard, K., 2007. Current amplification and magnetic reconnection at a 3D null point. Physical characteristics. J. Geophys. Res. 112, A03103.

Pontin, D.I., Galsgaard, K., Hornig, G., Priest, E.R., 2005a. A fully magnetohydrodynamic simulation of 3D non-null reconnection. Phys. Plasmas 12, 052307.

Pontin, D.I., Hornig, G., Priest, E.R., 2004. Kinematic reconnection at a magnetic null point: Spinealigned current. Geophys. Astrophys. Fluid Dynamics 98, 407-428.

Pontin, D.I., Hornig, G., Priest, E.R., 2005b. Kinematic reconnection at a magnetic null point: Fanaligned current. Geophys. Astrophys. Fluid Dynamics 99, 77-93.

Pontin, D.I., Wilmot-Smith, A.L., Hornig, G., Galsgaard, K., 2011. Dynamics of braided coronal loops. II. Cascade to multiple small-scale reconnection events. Astron. Astrophys. 525, A57.

Priest, E.R., Démoulin, P., 1995. Three-dimensional magnetic reconnection without null points. 1. Basic theory of magnetic flipping. J. Geophys. Res. 100, 23443-23463.

Priest, E.R., Forbes, T.G., 1992. Magnetic flipping - reconnection in three dimensions without null points. J. Geophys. Res. 97, 1521-1531.

Priest, E.R., Forbes, T.G., 2000. Magnetic reconnection: MHD theory and applications. Cambridge University Press, Cambridge.

Priest, E.R., Hornig, G., Pontin, D.I., 2003. On the nature of three-dimensional magnetic reconnection. J. Geophys. Res. 108, 1285.

Priest, E.R., Pontin, D.I., 2009. Three-dimensional null point reconnection regimes. Phys. Plasmas 16, 122101.

Priest, E.R., Titov, V.S., 1996. Magnetic reconnection at three-dimensional null points. Phil. Trans. R. Soc. A 354, 2951-2992.

Rappazzo, A.F., Velli, M., Einaudi, G., Dahlburg, R.B., 2008. Nonlinear dynamics of the Parker scenario for coronal heating. Astrophys. J. 677, 1348-1366.

Régnier, S., Parnell, C.E., Haynes, A.L., 2008. A new view of quiet-Sun topology from Hinode/SOT. Astron. Astrophys. 484, L47-L50.

Rickard, G.J., Titov, V.S., 1996. Current accumulation at a three-dimensional magnetic null. Astrophys. J. $472,840-852$. 
Schindler, K., Hesse, M., Birn, J., 1988. General magnetic reconnection, parallel electric fields, and helicity. J. Geophys. Res. 93, 5547-5557.

Titov, V.S., 2007. Generalized squashing factors for covariant description of magnetic connectivity in the solar corona. Astrophys. J. 660, 863-873.

Titov, V.S., Démoulin, P., 1999. Basic topology of twisted magnetic configurations in solar flares. Astron. Astrophys. 351, 707-720.

Titov, V.S., Forbes, T.G., Priest, E.R., Mikić, Z., Linker, J.A., 2009. Slip-squashing factors as a measure of three-dimensional magnetic reconnection. Astrophys. J. 693, 1029-1044.

Titov, V.S., Hornig, G., Démoulin, P., 2002. The theory of magnetic connectivity in the corona. J. Geophys. Res. 107, SSH 3-1.

Titov, V.S., Mikic, Z., Linker, J.A., Lionello, R., 2008. 1997 May 12 coronal mass ejection event. I. A simplified model of the preeruptive magnetic structure. Astrophys. J. 675, 1614-1628.

Titov, V.S., Tassi, E., Hornig, G., 2004. Exact solutions for steady reconnective annihilation revisited. Phys. Plasmas 11, 4662-4671.

Török, T., Aulanier, G., Schmieder, B., Reeves, K.K., Golub, L., 2009. Fan-spine topology formation through two-step reconnection driven by twisted flux emergence. Astrophys. J. 704, 485-495.

Ugarte-Urra, I., Warren, H.P., Winebarger, A.R., 2007. The magnetic topology of coronal mass ejection sources. Astrophys. J. 662, 1293-1301.

van Ballegooijen, A.A., 1985. Electric currents in the solar corona and the existence of magnetostatic equilibrium. Astrophys. J. 298, 421.

Wilmot-Smith, A.L., Hornig, G., Pontin, D.I., 2009a. Magnetic braiding and parallel electric fields. Astrophys. J. 696, 1339-1347.

Wilmot-Smith, A.L., Hornig, G., Pontin, D.I., 2009b. Magnetic braiding and quasi-separatrix layers. Astrophys. J. 704, 1288-1295.

Wilmot-Smith, A.L., Hornig, G., Priest, E.R., 2006. Dynamic non-null magnetic reconnection in three dimensions. I. Particular solutions. Proc. R. Soc. A 462, 2877-2895.

Wilmot-Smith, A.L., Hornig, G., Priest, E.R., 2009c. Dynamic non-null magnetic reconnection in three dimensions. II. Composite solutions. Geophys. Astrophys. Fluid Dynamics 103, 515-534.

Wilmot-Smith, A.L., Pontin, D.I., Hornig, G., 2010. Dynamics of braided coronal loops - I. Onset of magnetic reconnection. Astron. Astrophys. 516, A5.

Wyper, P., Jain, R., 2010. Torsional magnetic reconnection at three dimensional null points: A phenomenological study. Phys. Plasmas 17, 092902.

Xiao, C.J., Wang, X.G., Pu, Z.Y., et al., 2007. Satellite observations of separator-line geometry of three-dimensional magnetic reconnection. Nature Physics 3, 609-613.

Xiao, C.J., Wang, X.G., Pu, Z.Y., et al., 2006. In-situ evidence for the structure of the magnetic null in a 3D reconnection event in the Earth's magnetotail. Nature Physics 2, 478-483.

Yamada, M., Kulsrud, R., Ji, H., 2010. Magnetic reconnection. Reviews of Modern Physics 82, 603-664.

Zweibel, E.G., Yamada, M., 2009. Magnetic reconnection in astrophysical and laboratory plasmas. Ann. Rev. Astron. Astrophys. 47, 291-332. 